\title{
Tetraspanin CD151 mediates communication between PC3 prostate cancer cells and osteoblasts
}

\author{
Alicja Grudowska1, Dominika Czaplińska1,2, Wojciech Polom³, Marcin Matuszewski³, \\ Rafał Sądej ${ }^{\bowtie}$ and Andrzej C. Składanowski ${ }^{\bowtie}$ \\ 'Department of Molecular Enzymology, Intercollegiate Faculty of Biotechnology, University of Gdansk and Medical University of Gdansk, \\ Gdańsk, Poland; 2Department of Cell Biology, Intercollegiate Faculty of Biotechnology, University of Gdansk and Medical University of Gdansk, \\ Gdańsk, Poland; ${ }^{3}$ Department of Urology, Medical University of Gdansk, Gdańsk, Poland
}

Invasion and migration of cancer cells are crucial for the formation of secondary lesions. These require activation of signalling cascades modulated by the number of regulatory molecules. One such molecule is CD151, a member of evolutionary conserved tetraspanin family. CD151 is involved in cell adhesion, motility and cancer progression due to formation of complexes with laminin-binding integrins and regulation of growth factor receptors function (e.g. HGFR, TGF $\beta R$, EGFR). Recent studies point to correlation between CD151 expression and high tumour grade in prostate cancer $(\mathrm{PCa})$. Herein, we investigated a possible role of CD151 in communication between PC3 cancer cells and either cancer-associated fibroblasts (CAFs) or osteoblasts, an interplay which is significant for metastasis. The analysis showed that although CAFs strongly enhanced both migration and invasion of PC3 prostate cancer cells, the effect was not dependent on CD151. On the other hand, CD151 was found to promote 3D migration as well as invasive growth in response to osteoblasts-secreted growth factors. Obtained data revealed that knockdown of CD151 abolished activation of pro-migratory/pro-survival kinases (i.e FAK, Src, HSP27) triggered by osteoblasts, along with expression of matrix metalloproteinase-13. This suggests that CD151 participates in communication between PC3 cells and bone microenvironment and the process can be considered as a significant step of PCa progression and metastasis.

Key words: CD151, prostate cancer, osteoblasts

Received: 04 June, 2016; revised: 19 July, 2016; accepted: 26 August, 2016; available on-line: 14 March, 2017

e-mail: acskla@gumed.edu.pl (ACS), rsadej@gumed.edu.pl (RS) Abbreviations: a-SM-actin, a-smooth muscle-actin; CAFs, cancer-associated fibroblasts; ECM, extracellular matrix; FAK, focal adhesion kinase; FAP-1, fibroblast activation protein-1; hFOB, human fetal osteoblasts; HRP, horseradish peroxidase; HSP27, heat shock protein 27; MMP-13, matrix metalloproteinase 13; NHPF, norma human prostate fibroblasts; $\mathrm{PCa}$, prostate cancer; shRNA, short hairpin RNA

\section{INTRODUCTION}

Prostate cancer (PCa) affects $11 \%$ of male population mostly at its elderly age. Bone metastases in PCa contribute primarily to high mortality and morbidity of patients (Bubendorf et al., 2000). It is accepted that the development of carcinoma is strongly influenced by its microenvironment. In primary $\mathrm{PCa}$, stromal fibroblasts were shown to influence profoundly the transition from pre-invasive to invasive growth of tumor cells and are regarded as crucial and active participants of tumorigenesis and metastasis (Olumi et al., 1999). After homing in bone niche, tumour cells start to exert numerous paracrine interactions with hosting bone cells (osteoblasts, osteoclasts), bone marrow stem cells and hematopoietic progenitors. Such interplay leads to formation of osteoblastic (bone-forming) rather than osteoclastic (bone-resorbing) lesions in metastases, which indicates marked deregulation of bone formation (Roodman, 2004). Number of growth factors/regulatory peptides including bone morphogenetic proteins (BMPs), endothelins (ETs), fibroblast growth factors (FGFs) and others, were earlier correlated with increased osteoblastic activity (Logothetis \& Lin, 2005). Despite detailed characteristics of PCa cells action towards bone microenvironment deregulation (Hall et al., 2005), a reverse process, i.e. impact of bone microenvironment on PCa cells behaviour has not been fully understood.

Tetraspanins are characterized by the presence of four trans-membrane domains and are known to play crucial role in a number of physiological (proliferation, motility, cell adhesion etc.) and pathological conditions (tumour progression, metastasis) (reviewed in Sadej et al., 2014). Tetraspanin CD151 was the first member of this family associated with cancer progression. CD151 was found to promote invasion and metastasis in various in vitro and in vivo models of cancer (Chien et al., 2008; Kohno et al., 2002; Sadej et al., 2009; Sadej et al., 2010; Testa et al., 1999). High level of CD151 was proved to correlate with a poor prognosis for patients with several epithelial malignancies including lung (Tokuhara et al., 2001), breast (Kwon et al., 2012; Romanska et al., 2015b; Sadej et al., 2009; Yang et al., 2008), liver (Huang et al., 2010), colon (Hashida et al., 2003), pancreas (Zhu et al., 2011), and glioblastoma (Lee et al., 2013).

In prostate cancer, the expression of CD151 was found to be significantly higher in poorly differentiated PCa specimens and had a better prognostic value for predicting clinical outcome of $\mathrm{PCa}$ patients than the traditional histological grading (Gleason grading) (Ang et al., 2004). CD151 was shown to promote migration and invasion of PCa cells in vitro (Ang et al., 2010) and development of metastasis in experimental animal models of prostate cancer (Copeland et al., 2013; Detchokul et al., 2014). Whilst contribution of CD151 to cancer progression has been well established, it was also reported that in certain cell types the presence of CD151 is associated with suppression of both cell motility (Chien et al., 2008), tumour growth (Baldwin et al., 2014) and patients survival (Romanska et al., 2015a).

Functional interactions of CD151 with multiple proteins indispensable for cancer progression e.g. laminin-binding integrins (Kazarov et al., 2002; Sterk et al., 
2002; Yauch et al., 1998) or matrix metalloproteinases (Sugiura \& Berditchevski, 1999; Yanez-Mo et al., 2008) indicate mainly a modulatory role of this tetraspanin. Another feature of CD151 is associated with regulation of growth factors function, e.g. TGF- $\beta 1 / \mathrm{R}$ (Sadej et al., 2010), soluble growth factors secreted by endothelial cells (Sadej et al., 2009), HGF/R (Klosek et al., 2009) and EGF/R (Baldwin et al., 2008; Yang et al., 2008). This allows the estimation of CD151 contribution in modulation of activity of receptors for growth factors secreted by tumor microenvironment cells. Although few papers reported CD151 role in PCa progression (Ang et al., 2010; Copeland et al., 2013; Detchokul et al., 2014), the aspects of CD151 function in mediating signal from growth factors present in a niche of primary tumour and/or metastatic lesion and its consequences for PCa cells behaviour were never studied.

Herein, we focused on CD151 role in progression of PCa. Two distinct setups were assessed to determine CD151 role in communication between prostate cancer cells and primary tumour microenvironment - represented by cancer-associated fibroblasts - CAFs, or bone metastasis niche - represented by osteoblasts. PC3 cells derived from metastasis to the bone, therefore 'primed to invade and target skeleton, were used as a model in the study. CD151-regulated growth, migration and invasion were studied. Although there was no CD151-dependent effect, we found a very strong promotion of prostate cancer cell migration following exposure to CAFs-conditioned medium. On the other hand, CD151 was involved in migration and invasive growth of PC3 cells in the presence of growth factors secreted by osteoblasts. This seems to be related to CD151-dependent activation of FAK, Src and HSP27 as well as expression of MMP13. Therefore, CD151 function in regulation of prostate cancer cells metastasis to bones requires further studies.

\section{MATERIALS AND METHODS}

Cell lines, antibodies and reagents. PC3 - human prostate carcinoma cell line derived from bone metastasis and hFOB - conditionally immortalized human osteoblastic cell line were purchased from ATCC. PC3 cells were grown in DMEM medium supplemented with $10 \%$ fetal bovine serum and penicillin/streptomycin (100 U/ $\mathrm{ml}$ and $100 \mu \mathrm{g} / \mathrm{ml}$, respectively). hFOB cells were cultured in 1:1 mixture of Ham's F12 Medium and Dulbecco's Modified Eagle's Medium, with $2.5 \mathrm{mM}$ L-glutamine (without phenol red), penicillin/streptomycin and 10\% of fetal bovine serum. hFOB cells were grown either at $34^{\circ} \mathrm{C}$ (allowing rapid osteoblasts cell division) or at restrictive $39^{\circ} \mathrm{C}$ (resulting in cells differentiation towards more mature osteoblast phenotype). All media and their supplements were from Sigma-Aldrich. The following antibodies were obtained from Santa Cruz Biotechnology: anti-phospho-FAK (Tyr397) and anti-Src. Anti- $\beta$-actin, anti- $\alpha$-smooth muscle-actin, anti-CD151 (polyclonal), anti-FAP1 and anti-vimentin were from Sigma-Aldrich. Anti-phospho-Hsp27 (Ser82), anti-phospho-Src (Tyr416, produced by immunization of animals with a synthetic phosphopeptide corresponding to residues surrounding Tyr419 of human Src) and anti-MMP-13 were purchased from Cell Signaling Technology. Anti-CD151 antibody (5C11) used for cell sorting and flow cytometry was kindly donated by Dr. Fedor Berditchevski (University of Birmingham, UK). Secondary antibodies conjugated with AffiniPure DyLight 549 and AffiniPure DyLight 488 were from Jackson ImmunoResearch.
Isolation of fibroblasts from clinical material. Cancer-associated fibroblasts (CAFs) and normal human prostate fibroblasts (NHPFs) were isolated from adult patients (with advanced stage of $\mathrm{PCa}$ ) who underwent radical prostatectomy at Clinic of Urology, Medical University of Gdansk (approved by the Ethics Committee of the Medical University of Gdansk). CAFs were derived from neoplastic tissues whereas NHPFs from adjacent non-transformed tissue (confirmed by pathologist). Specimens were sliced into about $1 \times 1 \times 1 \mathrm{~mm}$ fragments and enzymatically digested with collagenase type I (225 $\mathrm{U} / \mathrm{ml}$ ) and hyaluronidase $(125 \mathrm{U} / \mathrm{ml}$ ) (Sigma-Aldrich) in complete medium with overnight rotation at $37^{\circ} \mathrm{C}$. Purified fibroblasts were used for further studies up to 6-8 passages. Conditioned medium was collected from fibroblast culture grown for minimum 2 days at high confluence.

Knockdown of CD151 in PC3 cells. CD151 silencing was performed with lentiviral transfer of shRNA targeting CD151 expression as previously described (Baldwin et al., 2008; Sadej et al., 2009) followed by cell sorting of CD151-negative population. Efficiency of knockdown was verified by Western blotting and flow cytometry analyses. As a control, PC3 cells were infected with virus carrying siRNA with a nonsense sequence.

Immunostaining. CAFs and NHPFs cells were fixed with $2 \%$ paraformaldehyde for 10 minutes and permeabilized with $0.1 \%$ Triton X-100 for 1 minute. After blocking with 3\% BSA solution, cells were washed with PBS and incubated with specific primary antibodies. Antibodies against FAP1 (1:1000), vimentin (1:5000) and $\alpha$-smooth muscle-actin $(1: 500)$ followed by appropriate secondary antibodies (coupled with AffiniPure DyLight 549 or DyLight 488, Jackson Immunoresearch) were used. Images were acquired with ZEISS AxioVision fluorescent microscope.

Western blotting. Cells grown up to $80-90 \%$ confluence were lysed with Laemmli buffer ( $2 \mathrm{x}$ concentrated) containing $2 \mathrm{mM}$ PMSF, $10 \mu \mathrm{g} / \mathrm{ml}$ aprotinin, $10 \mu \mathrm{g} /$ ml leupeptin, $5 \mathrm{mM}$ EGTA, $2 \mathrm{mM} \mathrm{Na} \mathrm{PO}_{7}, 5 \mathrm{mM} \mathrm{NaF}$ and $5 \mathrm{mM} \mathrm{Na}_{3} \mathrm{VO}_{4}$. Samples containing equal amounts of proteins per lane were loaded, resolved in SDSPAGE and then transferred onto nitrocellulose membrane. The membranes were blocked in 5\% skimmed milk and probed with specific antibodies overnight at $4^{\circ} \mathrm{C}$ followed by incubation with secondary antibodies conjugated with HRP (Sigma-Aldrich). $\beta$-actin was used as a protein load control. For signalling experiments cells were serum starved overnight and then stimulated with conditioned medium for indicated time points.

3-Dimensional cell cultures. $1.5 \times 10^{3}$ cells were resuspended in 1:1 growth factors-reduced matrigel (BD Biosciences) and standard culture medium. $40 \mu \mathrm{l}$ drops were placed in 12-well plates, incubated for 30-40 minutes in $37^{\circ} \mathrm{C}$ until matrigel solidified and then covered with $800 \mu \mathrm{l}$ of medium. For feeding experiments, conditioned medium (CM) from CAFs or osteoblasts was mixed 1:1 with DMEM. Cells were cultured in conditioned media up to 7-8 days. For morphological analyses of colonies 3D pictures were taken using a Zeiss Primovert microscope coupled with a camera (AxioCam ERc 5s, Zeiss). At least 50 representative colonies were included in morphometric analyses. Experiments were performed in triplicates. Comparative data were analysed with the unpaired Student's t-test using the Statistica 7.1 software.

Boyden Chamber Assay - analysis of cell transmigration or invasion. To analyse cell invasion, the lower compartment of inserts $(8-\mu \mathrm{m}$ pore size; BD Falcon) 
was coated with $30 \mu$ l of matrigel (diluted in DMEM to final concentration of $1 \mathrm{mg} / \mathrm{ml}$ ). In case of cell transmigration studies, the porous membrane of insert remained non-coated. Cells were detached with enzyme-free dissociation solution. $2 \times 10^{5}$ cells were dissolved in 400 $\mu l$ of serum-free DMEM and placed in upper chamber. Each well was filled with $1200 \mu$ l complete DMEM or DMEM/conditioned-medium (1:1). Nuclei of cells which migrated or invaded through matrigel-coated membrane after approximately $24 \mathrm{~h}$ were stained with Hoechst 33342 (Sigma Aldrich). Cells were counted under the ZEISS AxioVision fluorescent microscope. Comparative data were analysed with the unpaired Student's $t$-test using Statistica 7.1 software. Differences for which $p<0.01$ were considered as statistically significant.

Analysis of MMPs activity - zymography assay. Conditioned medium was collected from cells grown for $48 \mathrm{~h}$, centrifuged and supernatants were mixed with loading buffer (non-boiled, non-reduced samples). For a negative control, samples were dissolved in $2 \times$ Laemmli buffer with $\beta$-mercaptoethanol and boiled at $90^{\circ} \mathrm{C}$ for 10 minutes. All samples were applied on $8 \%$ resolving gel supplemented with gelatin type B $(0.1 \% \mathrm{w} / \mathrm{v})$ and separated in SDS-PAGE. Then gels were incubated in a renaturating buffer (containing 2.5\% Triton X-100) for 90 minutes to restore native structure of matrix metalloproteinases and left overnight in developing buffer (50 $\mathrm{mM}$ Tris- $\mathrm{HCl}, \mathrm{pH} 7.8,0.2 \mathrm{M} \mathrm{NaCl}, 5 \mathrm{mM} \mathrm{CaCl}$ and $0.02 \%$ Brij 35) which allows digestion of proteases substrates $\left(37^{\circ} \mathrm{C}\right)$. To visualise activity of MMPs (i.e. cleaved gelatine) gels were stained in Coomassie Brilliant Blue solution $(0.5 \%)$ followed by standard destaining procedure.

\section{RESULTS}

\section{Tetraspanin CD151 mediates PC3 cells migration and invasion but is not involved in CAF-driven effects}

Majority of cancer-associated fibroblasts originate from 'normal' residing fibroblasts but also from bone marrow-derived circulating cells, myeloid precursors as well as myofibroblasts activated during the process of tumorigenesis (reviewed in Bhowmick et al., 2004; Karagiannis et al., 2012; Tommelein et al., 2015). Cancer-asso-
A)

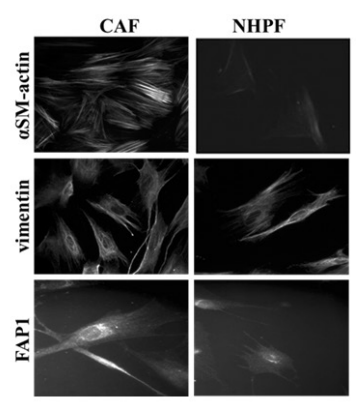

C)

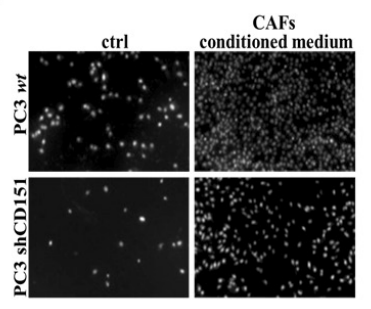

E)

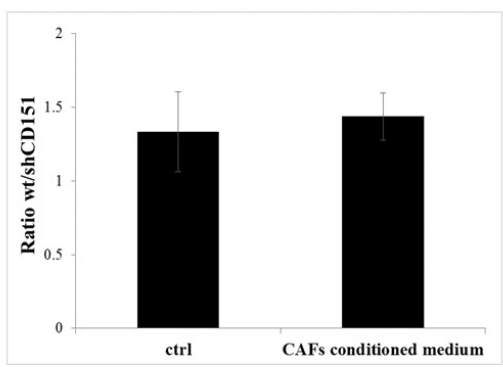

D)
B)
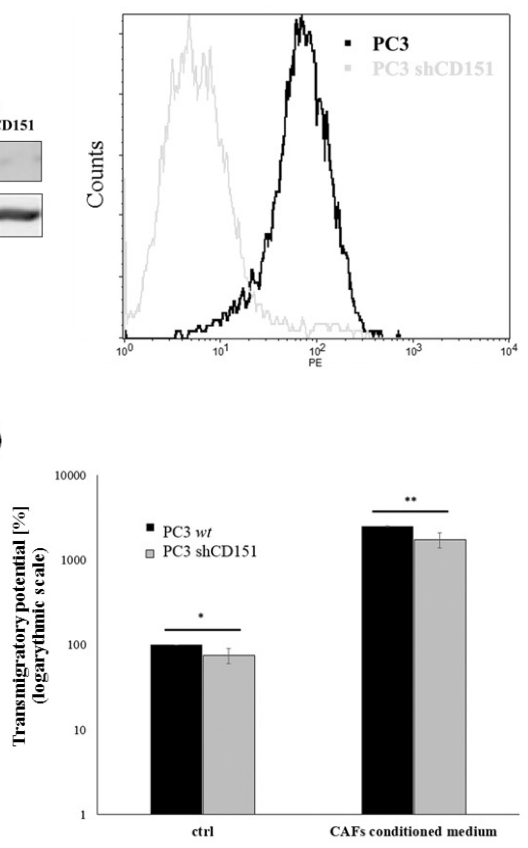

F)

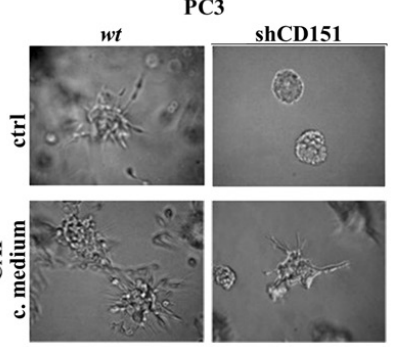

Figure 1. Analysis of CD151 involvement in CAFs impact on behaviour of PC3 cells.

(A) CAFs and NHPF were isolated from PCa patients. Cells were immunostained for a-SM-actin, vimentin and FAP-1 to verify their phenotype. (B) CD151 knockdown in PC3 cells was confirmed by Western blotting and flow cytometry. (C-E) Investigation of PC3 cells (wt vs. shCD151) transwell migration towards CAFs-conditioned media in Boyden chamber assay. Number of cells which migrated in control conditions was considered as $100 \%$. Data are presented as the mean \pm S.D., ${ }^{*} p<0.01,{ }^{* *} p<0.005$, (F) CD151-dependent PC3 cell growth in $3 \mathrm{D}$ matrigel \pm CAFs-conditioned medium. 
A)

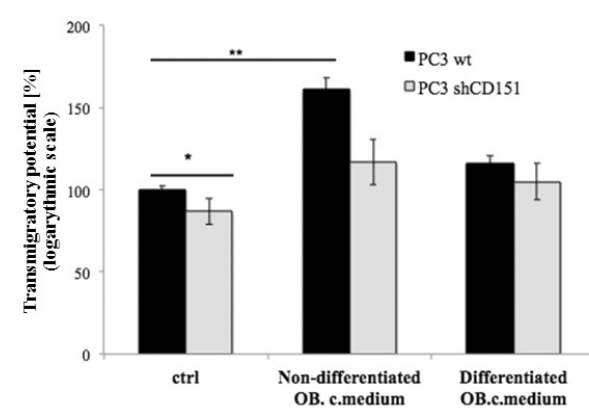

C)

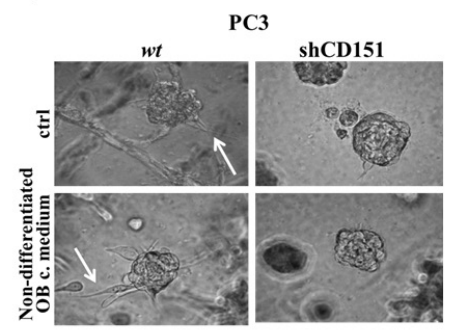

B)

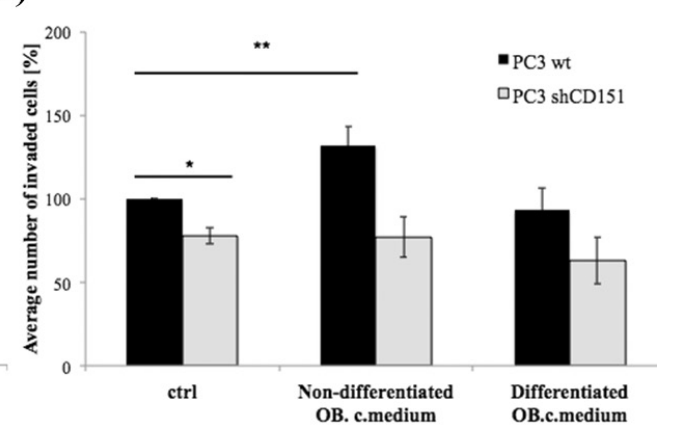

D)

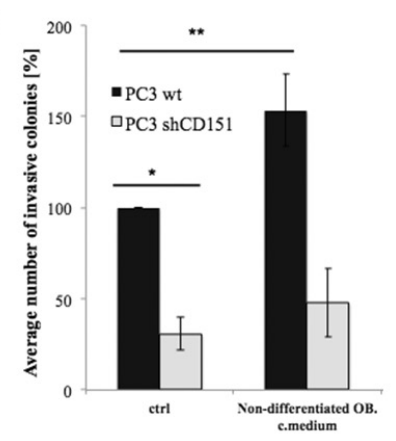

Figure 2. Analysis of CD151 involvement in osteoblasts impact on behaviour of PC3 cells.

Boyden chamber assay was performed in order to analyse PC3 cells (wt vs. shCD151) transwell migration $(\mathbf{A})\left({ }^{*} p<0.01,{ }^{* *} p<0.005\right)$ and invasion (B) $\left({ }^{*} p<0.006,{ }^{* *} p<0.001\right)$ towards osteoblasts-conditioned media (non-differentiated and differentiated). Number of cells which migrated/invaded through the membrane in control conditions was considered as $100 \%$. Data from three independent experiments are presented as the mean \pm S.D. (C) Cells were seeded in three-dimensional matrigel and cultured with/without hFOB (non-differentiated or differentiated)-conditioned media for 7-8 days. Representative pictures were taken under Zeiss Primovert Microscope (magnification 200x). (D) Quantification of invasive colonies number. Data from three independent experiments are presented as the mean \pm S.D., ${ }^{*} p<0.001,{ }^{* *} p<0.003$.

ciated fibroblasts express all markers of myofibroblasts (including alpha SM-actin) (Bhowmick et al., 2004; Karagiannis et al., 2012). In the vast majority of papers the term 'CAF' involves all fractions of fibroblasts present in tumor's reactive stroma and is actually interchangeable with the term 'activated myofibroblasts` (De Wever et al., 2008; Karagiannis et al., 2012; Tommelein et al., 2015). A series of excellent papers clearly suggests involvement of activated myofibroblasts/CAFs in PCa progression (Comito et al., 2014; Liao et al., 2010; Olumi et al., 1999; Taddei et al., 2014). To analyse impact of cancer-associated fibroblasts on motility and invasion of PC3 prostate cancer cell, a set of few primary fibroblast cell lines was established from cancerous and adjacent tissue samples taken from radical prostatectomies of PCa patients. CAFs were confirmed to express specific, previously reported markers (Garin-Chesa et al., 1990) i.e. elevated expression of $\alpha$-SM-actin ( $\alpha$-smooth muscle-actin) and FAP-1 (Fibroblasts Activation Protein-1) which distinguishes them from normal fibroblasts (Fig. 1A). The study itself was focused on elucidating the CD151 involvement in mediating communication between prostate cancer cells and cancer-associated fibroblasts - crucial contributors of the progression of the disease. To this end CD151-negative variant of PC3 cells was generated by transfer of specific shRNA. High efficiency of CD151 knockdown (over 95\%) was assessed by Western blot and flow cytometry (Fig. 1B). Analysis of cell migration in control conditions showed that depletion of CD151 in PC3 cells impaired their transwell migratory potential by over 25\% $(p<0.01)$ (Fig. 1C-D). Importantly, CAF-conditioned medium (containing all secreted growth factors) had a profound impact on PC3 cells migration, giving over 35 -fold increase in a number of migrated cells. Due to tremendous promotion of cell migration in response to CAFs-conditioned medium, the results of experiments are presented in logarithmic scale (Fig. 1D). Noteworthy, calculation of PC3 (CD151-positive)/PC3 shCD151 cell number ratio revealed that depletion of CD151 reduced cell migration down to similar extent in both experimental setups (control vs. CAF-conditioned medium) (Fig. 1E). Further analysis demonstrated that PC3 cells formed invasive 3D matrigel-penetrating colonies and such phenotype was abolished by knockdown of CD151 (Fig. 1F). CAFs conditioned medium enhanced invasive properties of PC3 cells which was also impaired following CD151-silencing. Despite promotion of invasiveness, there was no significant impact on those colonies size or cells proliferation (in both $2 \mathrm{D}$ and $3 \mathrm{D}$ conditions, data not shown). The results clearly show that although CD151 participates in PC3 cells migration and invasion, it does not take part in those activities driven by cancerassociated fibroblasts.

\section{CD151 facilitates osteoblast-driven PC3 migratory and invasive phenotype}

Osteoblasts contribution to bone metastasis of prostate cancer was previously suggested (Roodman, 2004). PC3 cells transwell migration and invasion were analysed towards both non-differentiated and differentiated human fetal osteoblasts (hFOB)-conditioned media to determine CD151 function in putative osteoblasts impact on these activities. Firstly, we found that knock-down of CD151 affected not only cell migration but also cell invasion (Fig. 2A-B). Secondly, non-differentiated osteoblasts were shown to stimulate both transwell migration $(\sim 1.61 \mathrm{x})$ and invasion $(\sim 1.32 \times)$ of PC3 cells (Fig. 2A 
and 2B) in CD151-dependent manner. Surprisingly there was no influence of growth factors/cytokines secreted by differentiated osteoblasts on the behaviour of PC3 cells. Further analyses, focused on pattern of PC3 cells 3D matrigel growth, confirmed promotion of invasive phenotype by non-differentiated hFOB (Fig. 2C). Although colonies formed by PC3 cells displayed invasive protrusions, their length was greater in response to signal from osteoblasts (due to lack of differentiated hFOBs impact in transwell migration/invasion assays here we tested only non-differentiated hFOBs). In addition, the number of invasive colonies was larger following exposure to osteoblasts-conditioned medium (Fig. 2D). As expected CD151-silencing strongly affected impact of growth factors secreted by osteoblasts on PC3 cells type of growth (Fig. 2C and 2D).

\section{CD151 is involved in regulation of promigratory and proinvasive signalling pathways in response to osteoblasts-secreted growth factors}

Our next aim was to define molecular mechanism by which CD151 might promote migration or invasion following stimuli from osteoblasts. According to the literature, CD151 can influence growth factors-mediated communication with the tumour microenvironment only when it is in a complex with integrins (Deng et al., 2012; Franco et al., 2010; Sadej et al., 2009; Sadej et al., 2010). PC3 cells with various status of CD151 were exposed to hFOBsconditioned media and analysed for activation of FAK, Src (main mediators of integrins-triggered signalling) and HSP27 (chosen due to profound contribution to PCa progression shown in vitro, in vivo (Voll et al., 2014) and in clinical studies (Cornford et al., 2000)). Under stimulation by non-differentiated osteoblasts-conditioned medium, a marked increase in phosphorylation of these promigratory kinases was observed (Fig. 3A). As expected, knockdown of CD151 strongly attenuated activation of FAK, Src and HSP27. FAK/Src are direct, fairly upstream mediators of integrin- and/or growth factor receptors-triggered signalling whereas HSP27 is a downstream effector of p38 kinase. Therefore it is not surprising that kinetics of FAK/
Src vs. HSP27 in CD151-dependent activation is different. CD151 involvement in regulation of these kinases following exposure to osteoblasts-condtioned media can be linked directly to the observed differences in cell migration (Fig. 2A). Invasive behaviour of cancer cells requires activity of matrix metalloproteinases, capable of degrading ECM proteins. These enzymes were found to be regulated by CD151 according to few studies (Hong et al., 2006; Yue et al., 2013). In order to examine contribution of CD151 to the control of matrix metalloproteinases activity upon osteoblasts stimulation, zymography assay was performed. We found that increased invasive behaviour of PC3 cells following their exposure to osteoblasts-conditioned medium correlated with the elevated activity of ECM-degrading matrix metalloproteinase (Fig. 3B). The obtained zymogram showed that although there was no bands associated with function of MMP-2 (72 kDa) or MMP-9 (92 $\mathrm{kDa}$ ) (both shown to be regulated by CD151 (Yanez-Mo et al., 2008; Zhang et al., 2016)), a clear CD151-dependent digestive activity, located at molecular weight of approx. $48 \mathrm{kDa}$, typical for MMP-13 was observed (Fig. 3B). This was elevated following treatment with hFOBs-conditioned media, primarily in PC3 (CD151-positive) cells. Analysis of MMP-13 expression in PC3 vs. PC3 shCD151 cells revealed engagement of tetraspanin in regulation of MMP13 level (Fig. 3C). Moreover, cells treatment with non-differentiated osteoblasts-conditioned media resulted in enhanced MMP-13 expression in CD151-dependent manner. Therefore we conclude that promotion of PC3 cells invasion in response to signal from osteoblasts depends on CD151-driven expression rather than activity of MMP-13.

\section{DISCUSSION}

The occurrence of prostate cancer is estimated as the second most frequent disease in elderly male population. Despite enormous effort, which has been put in understanding the mechanism governing PCa development, the specificity of the routine prognostic approaches is still raising controversies (Collette et al., 2006). Therefore, the primary aim of this study was to investigate

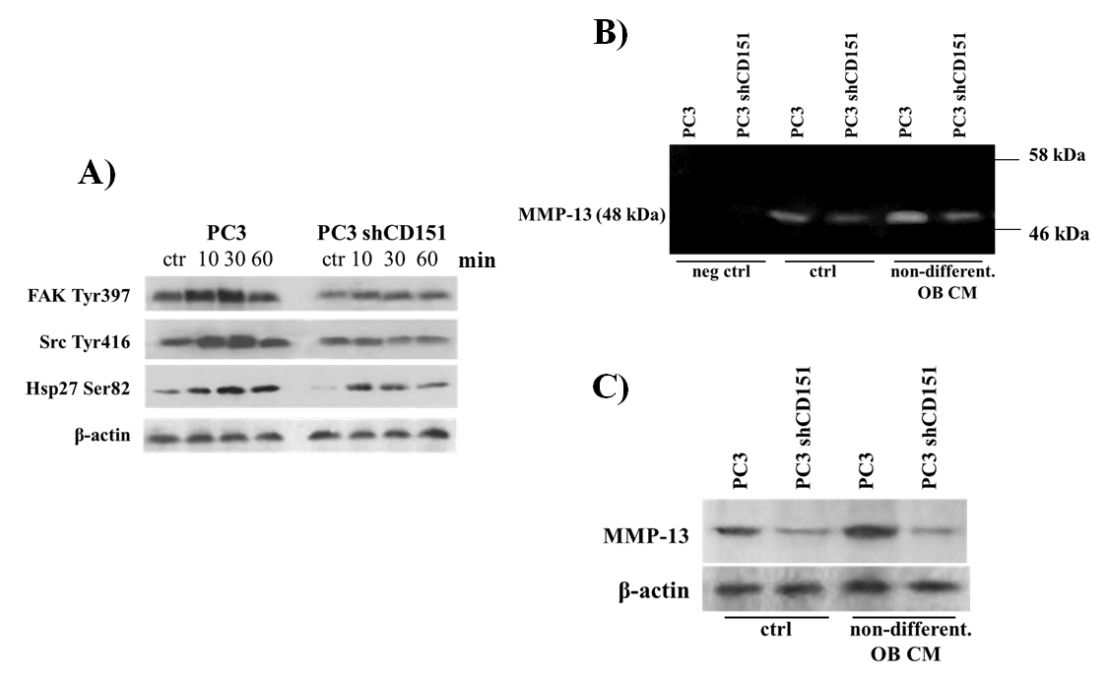

Figure 3. Osteoblasts activate pro-migratory signalling pathway and expression of MMP-13 in CD151-dependent manner.

(A) PC3 cells were starved overnight and stimulated with osteoblasts-conditioned media for indicated time points (10, 30,60 minutes). Activation of specific proteins was analysed by Western blotting as described in Materials and Methods. (B) PC3 cells were seeded in equal numbers on $60 \mathrm{~mm}$ plates and incubated with osteoblast conditioned-medium for $48 \mathrm{~h}$ prior overnight starvation. Samples of media with secreted MMPs collected from cell culture media were analysed by zymography for proteolytic activity of MMPs. (C) Detection of MMP-13 in PC3 wt vs. PC3 shCD151 \pm osteoblasts-conditioned media. All experiments were reproducible. 
possible role of one candidate - tetraspanin CD151 in PCa progression and metastasis.

Previous reports suggested CD151 function in communication with tumour microenvironments at both primary tumour and distant metastases (Franco et al., 2010; Klosek et al., 2005; Sadej et al., 2009; Sadej et al., 2010). Herein we analysed CD151 involvement in the behaviour of prostate cancer cells exposed to growth factors secreted by CAFs (derived from primary tumour) or osteoblasts (representing bone niche). We found that silencing of CD151 impaired both migration and invasion of PC3 cells. On the other hand CAFs-conditioned medium vastly promoted cell motility and 3D invasion without obvious dependence on CD151 status (Fig. 1C-F). Therefore, we conclude that tumour fibroblasts undoubtedly promote prostate cancer cell migration and invasion (probably contributing to their escape from the primary site) however CD151 per se does not participate in these events. In the course of the study, the interplay between PC3 cells and osteoblasts (representing bone niche) was also analysed. There was no clear effect of differentiated osteoblasts on cancer cell migration and invasion which suggests lack of motility/ invasion-promoting growth factors secreted by this pool of osteoblasts. On the other hand, non-differentiated, actively proliferating osteoblasts did enhance activity of both tested PC3 cells. Importantly, CD151 exerted clear growth and invasion promoting effect (at Boyden chamber and 3D culture systems) (Fig. 2A-D). CD151 was previously proved to regulate motility and invasion of PC3 cells (Ang et al., 2010), however our finding suggests that these process can be additionally enhanced by osteoblasts-secreted growth factors in CD151-dependent manner. Certainly, it would be valuable to analyse CD151 function in response of androgen-sensitive PCa cell lines (e.g. LnCap). This approach could define a potential function of CD151 in early steps of PCa progression.

It was reported that promigratory function of CD151 in PC3 cells was based on regulation of ERK activity (Yang et al., 2012). We additionally showed that presence of CD151 facilitates phosphorylation of FAK, Src and HSP27 (considered as directly involved in regulation of cell migration) in response to osteoblasts-conditioned medium. In addition, we analysed mechanism of CD151-promoted cell invasion following stimulation originating from osteoblasts. CD151-positive PC3 cells were characterised by higher activity of MMP-13 than CD151-negative cells and the difference was enhanced by osteoblasts action. We proved that it was based on CD151-regulated expression, rather than activity, of MMP-13. Limited information concerning osteoblasts influence on PC3 cells has been so far reported. This interaction was studied i.e. in mineralized human primary osteoblasts matrices which stimulated acquisition of invasive phenotype and proliferation of PC3 cells (Reichert et al., 2010). Others showed that coculturing of both cell types in tissue-engineered bone construct elevated MMP-9 level (Sieh et al., 2010). These data are in agreement with our studies showing osteoblasts-dependent promotion of prostate cancer cells migration and invasion and involvement of CD151 in regulation of these processes. The interdependence between CD151 and MMP13 was previously shown in rat pancreatic carcinoma (Yue et al., 2013). Our finding that CD151 participates in regulation of MMP-13 expression in response to bone niche stimuli, seems to be particularly important in the context of MMPs significance in extracellular matrix remodelling during formation of PCa metastases (Hornebeck et al., 2002; Nemeth et al., 1999).

Certainly, it would be tempting to identify growth factor(s) secreted by osteoblasts whose function is regulated by CD151 in PCa cells. One of candidate is TGF- $\beta 1$ proved to mediate chemotaxis of PC3 cells after treatment with osteoblasts-conditioned medium (Festuccia et al., 1999). Although CD151 was shown to regulate TGF- $\beta 1$-driven lung metastasis of breast cancer (Sadej et al., 2010) we did not manage to identify CD151 involvement in TGF- $\beta 1$ in osteoblasts-triggered PC3 cells migration or invasion (data not shown). This aspect i.e. a putative mediation of growth factors-based stimuli from bone niche by CD151 and subsequent consequences for metastasis requires further studies.

\section{Acknowledgements}

The authors are grateful to Dr Fedor Berditchevski (University of Birmingham, UK) for antibody and plasmid sharing.

\section{Acknowledgements}

This work was supported by research grants PRELUDIUM 3 (ID No. 2012/05/N/NZ3/00290) from National Science Center and HOMING PLUS (2010-2/12) from The Foundation for Polish Science.

\section{REFERENCES}

Ang J, Fang B-L, Ashman LK, Frauman AG (2010) The migration and invasion of human prostate cancer cell lines involves CD151 expression. Oncology Reports 24. Doi: 10.3892/or_00001022

Ang J, Lijovic M, Ashman LK, Kan K, Frauman AG (2004) CD151 protein expression predicts the clinical outcome of low-grade primary prostate cancer better than histologic grading: a new prognostic indicator? Cancer Epidemiol Biomarkers Prev 13: 1717-1721.

Baldwin G, Novitskaya V, Sadej R, Pochec E, Litynska A, Hartmann C, Williams J, Ashman L, Eble JA, Berditchevski F (2008) Tetraspanin CD151 regulates glycosylation of (alpha)3(beta) 1 integrin. $J$ Biol Chem 283: 35445-35454. doi: 10.1074/jbc.M806394200

Baldwin LA, Hoff JT, Lefringhouse J, Zhang M, Jia C, Liu Z, Erfani S, Jin $\mathrm{H}$, Xu M, She QB, van Nagell JR, Wang C, Chen L, Plattner R, Kaetzel DM, Luo J, Lu M, West D, Liu C, Ueland FR, Drapkin R, Zhou BP, Yang XH (2014) CD151- $\alpha 3 \beta 1$ integrin complexes suppress ovarian tumor growth by repressing slug-mediated EMT and canonical Wnt signaling. Oncotarget 5: 12203-12217. doi: 10.18632/ oncotarget. 2622

Bhowmick NA, Neilson EG, Moses HL (2004) Stromal fibroblasts in cancer initiation and progression. Nature 432: 332-337. doi: 10.1038 / nature03096

Bubendorf L, Schöpfer A, Wagner U, Sauter G, Moch H, Willi N, Gasser TC, Mihatsch MJ (2000) Metastatic patterns of prostate cancer: an autopsy study of 1,589 patients. Hum Pathol 31: 578-583.

Chien CW, Lin SC, Lai YY, Lin BW, Lee JC, Tsai SJ (2008) Regulation of CD151 by hypoxia controls cell adhesion and metastasis in colorectal cancer. Clin Cancer Res 14: 8043-8051. doi: 10.1158/10780432.CCR-08-1651

Collette L, Burzykowski T, Schröder FH (2006) Prostate-specific antigen (PSA) alone is not an appropriate surrogate marker of longterm therapeutic benefit in prostate cancer trials. Eur J Cancer 42: 1344-1350. doi: 10.1016/j.ejca.2006.02.011

Comito G, Giannoni E, Segura CP, Barcellos-de-Souza P, Raspollini MR, Baroni G, Lanciotti M, Serni S, Chiarugi P (2014) Cancerassociated fibroblasts and M2-polarized macrophages synergize during prostate carcinoma progression. Oncogene 33: 2423-2431. doi: 10.1038/onc. 2013.191

Copeland BT, Bowman MJ, Ashman LK (2013) Genetic ablation of the tetraspanin CD151 reduces spontaneous metastatic spread of prostate cancer in the TRAMP model. Mol Cancer Res 11: 95-105. doi: 10.1158/1541-7786.MCR-12-0468

Cornford PA, Dodson AR, Parsons KF, Desmond AD, Woolfenden A, Fordham M, Neoptolemos JP, Ke Y, Foster CS (2000) Heat shock protein expression independently predicts clinical outcome in prostate cancer. Cancer Res 60: 7099-7105.

De Wever O, Demetter P, Mareel M, Bracke M (2008) Stromal myofibroblasts are drivers of invasive cancer growth. Int J Cancer 123: 2229-2238. doi: 10.1002/ijc.23925

Deng X, Li Q, Hoff J, Novak M, Yang H, Jin H, Erfani SF, Sharma C, Zhou P, Rabinovitz I, Sonnenberg A, Yi Y, Stipp CS, Kaetzel DM, Hemler ME, Yang XH (2012) Integrin-associated CD151 drives ErbB2-evoked mammary tumor onset and metastasis. Neoplasia 14: 678-689. doi: 10.1593/neo.12922 
Detchokul S, Newell B, Williams ED, Frauman AG (2014) CD151 is associated with prostate cancer cell invasion and lymphangiogenesis in vivo. Oncol Rep 31: 241-247. doi: 10.3892/or.2013.2823

Festuccia C, Bologna M, Gravina GL, Guerra F, Angelucci A, Villanova I, Millimaggi D, Teti A (1999) Osteoblast conditioned media contain TGF-beta1 and modulate the migration of prostate tumor cells and their interactions with extracellular matrix components. Int J Cancer 81: 395-403. doi: 10.1002/(SICI)10970215(19990505)81:3<395::AID-IJC13>3.0.CO;2-V

Franco M, Muratori C, Corso S, Tenaglia E, Bertotti A, Capparuccia L, Trusolino L, Comoglio PM, Tamagnone L (2010) The tetraspanin CD151 is required for Met-dependent signaling and tumor cell growth. J Biol Chem 285: 38756-38764. doi: 10.1074/jbc. M110.145417

Garin-Chesa P, Old LJ, Rettig WJ (1990) Cell surface glycoprotein of reactive stromal fibroblasts as a potential antibody target in human epithelial cancers. Proc Natl Acad Sci U S A 87: 7235-7239.

Hall CL, Bafico A, Dai J, Aaronson SA, Keller ET (2005) Prostate cancer cells promote osteoblastic bone metastases through Wnts. Cancer Res 65: 7554-7560. doi: 10.1158/0008-5472.CAN-05-1317

Hashida H, Takabayashi A, Tokuhara T, Hattori N, Taki T, Hasegawa H, Satoh S, Kobayashi N, Yamaoka Y, Miyake M (2003) Clinical significance of transmembrane 4 superfamily in colon cancer. $\mathrm{Br} J$ Cancer 89: 158-167. doi: 10.1038/sj.bjc.6601015

Hong IK, Jin YJ, Byun HJ, Jeoung DI, Kim YM, Lee H (2006) Homophilic interactions of Tetraspanin CD151 up-regulate motility and matrix metalloproteinase- 9 expression of human melanoma cells through adhesion-dependent c-Jun activation signaling pathways. J Biol Chem 281: 24279-24292. doi: 10.1074/jbc.M601209200

Hornebeck W, Emonard H, Monboisse JC, Bellon G (2002) Matrixdirected regulation of pericellular proteolysis and tumor progression. Semin Cancer Biol 12: 231-241.

Huang XY, Ke AW, Shi GM, Ding ZB, Devbhandari RP, Gu FM, Li QL, Dai Z, Zhou J, Fan J (2010) Overexpression of CD151 as an adverse marker for intrahepatic cholangiocarcinoma patients. Cancer 116: 5440-5451. doi: $10.1002 /$ cncr.25485

Karagiannis GS, Poutahidis T, Erdman SE, Kirsch R, Riddell RH, Diamandis EP (2012) Cancer-associated fibroblasts drive the progression of metastasis through both paracrine and mechanical pressure on cancer tissue. Mol Cancer Res 10: 1403-1418. doi: 10.1158/15417786.MCR-12-0307

Kazarov AR, Yang X, Stipp CS, Sehgal B, Hemler ME (2002) An extracellular site on tetraspanin CD151 determines alpha 3 and alpha 6 integrin-dependent cellular morphology. J Cell Biol 158: 12991309. doi: $10.1083 /$ jcb. 200204056

Klosek SK, Nakashiro K, Hara S, Goda H, Hasegawa H, Hamakawa H (2009) CD151 regulates HGF-stimulated morphogenesis of human breast cancer cells. Biochem Biophys Res Commun 379: 1097-1100. doi: 10.1016/j.bbrc.2009.01.023

Klosek SK, Nakashiro K, Hara S, Shintani S, Hasegawa H, Hamakawa H (2005) CD151 forms a functional complex with c-Met in human salivary gland cancer cells. Biochem Biophys Res Commun 336: 408-416. doi: $10.1016 /$ j.bbrc.2005.08.106

Kohno M, Hasegawa H, Miyake M, Yamamoto T, Fujita S (2002) CD151 enhances cell motility and metastasis of cancer cells in the presence of focal adhesion kinase. Int J Cancer 97: 336-343. doi: 10.1002/ijc.1605

Kwon MJ, Park S, Choi JY, Oh E, Kim YJ, Park YH, Cho EY, Nam SJ, Im YH, Shin YK, Choi YL (2012) Clinical significance of CD151 overexpression in subtypes of invasive breast cancer. $\mathrm{Br} J$ Cancer 106: 923-930. doi: 10.1038/bjc.2012.11

Lee D, Suh YL, Park TI, Do IG, Seol HJ, Nam DH, Kim ST (2013) Prognostic significance of tetraspanin CD151 in newly diagnosed glioblastomas. J Surg Oncol 107: 646-652. doi: 10.1002/jso.23249

Liao CP, Adisetiyo H, Liang M, Roy-Burman P (2010) Cancer-associated fibroblasts enhance the gland-forming capability of prostate cancer stem cells. Cancer Res 70: 7294-7303. doi: 10.1158/0008-5472. CAN-09-3982

Logothetis CJ, Lin SH (2005) Osteoblasts in prostate cancer metastasis to bone. Nat Rev Cancer 5: 21-28. doi: 10.1038/nrc1528

Nemeth JA, Harb JF, Barroso U, He Z, Grignon DJ, Cher ML (1999) Severe combined immunodeficient-hu model of human prostate cancer metastasis to human bone. Cancer Res 59: 1987-1993.

Olumi AF, Grossfeld GD, Hayward SW, Carroll PR, Tlsty TD, Cunha GR (1999) Carcinoma-associated fibroblasts direct tumor progression of initiated human prostatic epithelium. Cancer Res 59: 50025011.

Reichert JC, Quent VM, Burke LJ, Stansfield SH, Clements JA, Hutmacher DW (2010) Mineralized human primary osteoblast matrices as a model system to analyse interactions of prostate cancer cells with the bone microenvironment. Biomaterials 31: 7928-7936. doi: 10.1016/j.biomaterials.2010.06.055

Romanska HM, Potemski P, Krakowska M, Mieszkowska M, Chaudhri S, Kordek R, Kubiak R, Speirs V, Hanby AM, Sadej R, Berditchevski F (2015a) Lack of CD151/integrin $\alpha 3 \beta 1$ complex is predictive of poor outcome in node-negative lobular breast carcinoma: oppos- ing roles of CD151 in invasive lobular and ductal breast cancers. $\mathrm{Br}$ J Cancer 113: 1350-1357. doi: 10.1038/bjc.2015.344

Romanska HM, Potemski P, Kusinska R, Kopczynski J, Sadej R, Kordek R (2015b) Expression of CD151/Tspan24 and integrin alpha 3 complex in aid of prognostication of HER2-negative highgrade ductal carcinoma in situ. Int J Clin Exp Pathol 8: 9471-9478.

Roodman GD (2004) Mechanisms of bone metastasis. N Engl J Med 350: 1655-1664. doi: 10.1056/NEJMra030831

Sadej R, Grudowska A, Turczyk L, Kordek R, Romanska HM (2014) CD151 in cancer progression and metastasis: a complex scenario. Lab Invest 94: 41-51. doi: 10.1038/labinvest.2013.136

Sadej R, Romanska H, Baldwin G, Gkirtzimanaki K, Novitskaya V, Filer AD, Krcova Z, Kusinska R, Ehrmann J, Buckley CD, Kordek R, Potemski P, Eliopoulos AG, Lalani e-N, Berditchevski F (2009) CD151 regulates tumorigenesis by modulating the communication between tumor cells and endothelium. Mol Cancer Res 7: 787-798. doi: 10.1158/1541-7786.MCR-08-0574

Sadej R, Romanska H, Kavanagh D, Baldwin G, Takahashi T, Kalia N, Berditchevski F (2010) Tetraspanin CD151 regulates transforming growth factor beta signaling: implication in tumor metastasis. Cancer Res 70: 6059-6070. doi: 10.1158/0008-5472.CAN-09-3497

Sieh S, Lubik AA, Clements JA, Nelson CC and Hutmacher DW (2010) Interactions between human osteoblasts and prostate cancer cells in a novel 3D in vitro model. Organogenesis 6: 181-188.

Sterk LM, Geuijen CA, van den Berg JG, Claessen N, Weening JJ, Sonnenberg A (2002) Association of the tetraspanin CD151 with the laminin-binding integrins alpha3beta1, alpha6beta1, alpha6beta4 and alpha7beta1 in cells in culture and in vivo. J Cell Sci 115: 11611173.

Sugiura T, Berditchevski F (1999) Function of alpha3beta1-tetraspanin protein complexes in tumor cell invasion. Evidence for the role of the complexes in production of matrix metalloproteinase 2 (MMP2). J Cell Biol 146: 1375-1389. doi: 10.1083/jcb.146.6.1375

Taddei ML, Cavallini L, Comito G, Giannoni E, Folini M, Marini A, Gandellini P, Morandi A, Pintus G, Raspollini MR, Zaffaroni N, Chiarugi P (2014) Senescent stroma promotes prostate cancer progression: the role of miR-210. Mol Oncol 8: 1729-1746. doi: 10.1016/j.molonc.2014.07.009

Testa JE, Brooks PC, Lin JM, Quigley JP (1999) Eukaryotic expression cloning with an antimetastatic monoclonal antibody identifies a tetraspanin (PETA-3/CD151) as an effector of human tumor cell migration and metastasis. Cancer Res 59: 3812-3820.

Tokuhara T, Hasegawa H, Hattori N, Ishida H, Taki T, Tachibana S, Sasaki S, Miyake M (2001) Clinical significance of CD151 gene expression in non-small cell lung cancer. Clin Cancer Res 7: 4109-4114.

Tommelein J, Verset L, Boterberg T, Demetter P, Bracke M, De Wever O (2015) Cancer-associated fibroblasts connect metastasis-promoting communication in colorectal cancer. Front Oncol 5: 63. Doi: 10.3389 / fonc. 2015.00063

Voll EA, Ogden IM, Pavese JM, Huang X, Xu L, Jovanovic BD, Bergan RC (2014) Heat shock protein 27 regulates human prostate cancer cell motility and metastatic progression. Oncotarget 5: 2648-2663. doi: 10.18632/oncotarget.1917

Yanez-Mo M, Barreiro O, Gonzalo P, Batista A, Megias D, Genis L, Sachs N, Sala-Valdes M, Alonso MA, Montoya MC, Sonnenberg A, Arroyo AG, Sanchez-Madrid F (2008) MT1-MMP collagenolytic activity is regulated through association with tetraspanin CD151 in primary endothelial cells. Blood 112: 3217-3226. doi: 10.1182/ blood-2008-02-139394

Yang W, Li P, Lin J, Zuo H, Zuo P, Zou Y, Liu Z (2012) CD151 promotes proliferation and migration of PC3 cells via the formation of CD151-integrin $\alpha 3 / \alpha 6$ complex. I Huazhong Univ Sci Technolog Med Sci 32: 383-388. doi: 10.1007/s11596-012-0066-y

Yang XH, Richardson AL, Torres-Arzayus MI, Zhou P, Sharma C, Kazarov AR, Andzelm MM, Strominger JL, Brown M, Hemler ME (2008) CD151 accelerates breast cancer by regulating alpha 6 integrin function, signaling, and molecular organization. Cancer Res 68: 3204-3213. doi: 10.1158/0008-5472.CAN-07-2949

Yauch RL, Berditchevski F, Harler MB, Reichner J, Hemler ME (1998) Highly stoichiometric, stable, and specific association of integrin alpha3beta1 with CD151 provides a major link to phosphatidylinositol 4-kinase, and may regulate cell migration. Mol Biol Cell 9: 27512765. doi: $10.1091 / \mathrm{mbc} .9 .10 .2751$

Yue S, Mu W, Zöller M (2013) Tspan8 and CD151 promote metastasis by distinct mechanisms. Eur J Cancer 49: 2934-2948. doi: 10.1016/j. ejca.2013.03.032

Zhang Z, Wang F, Li Q, Zhang H, Cui Y, Ma C, Zhu J, Gu X, Sun Z (2016) CD151 knockdown inhibits osteosarcoma metastasis through the GSK-3beta/beta-catenin/MMP9 pathway. Oncol Rep 35: 1764 1770. doi: $10.3892 /$ or.2015.4517

Zhu GH, Huang C, Qiu ZJ, Liu J, Zhang ZH, Zhao N, Feng ZZ, Lv XH (2011) Expression and prognostic significance of CD151, c-Met, and integrin alpha3/alpha6 sin pancreatic ductal adenocarcinoma. Dig Dis Sci 56: 1090-1098. doi: 10.1007/s10620-010-1416-x 